
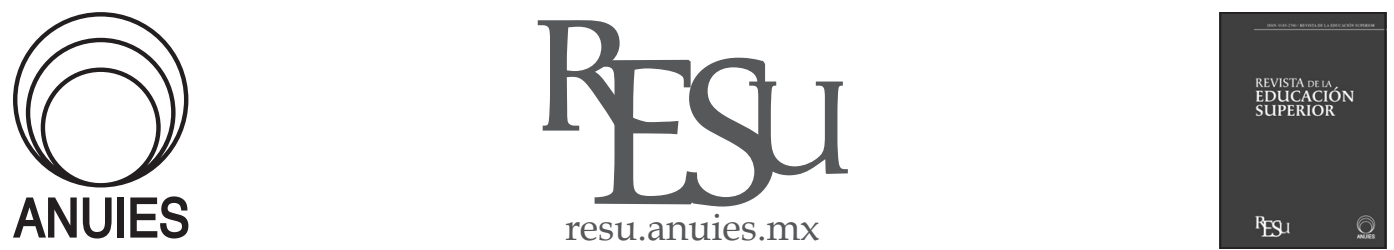

Revista de la Educación Superior 47 (188) (2018)

ARTíCULO

\title{
¿Y esto para qué me va a servir? Jóvenes universitarios y literacidades investigativas*
}

\section{Pendiente}

\section{Claudia Salinas Boldo**, María Guadalupe Tinajero Villavicencio ${ }^{* * *}$ y Eyder Gabriel Sima Lozano**** $^{* * 2}$}

\footnotetext{
* Agradecemos al Programa para el Desarrollo Profesional Docente para el Tipo Superior, de la Dirección de Superación Académica de la Secretaría de Educación Pública, por los recursos brindados para llevar a cabo el presente proyecto, en la forma de Apoyo a la Incorporación de Nuevos Profesores de Tiempo Completo Convocatoria 2016.

A todos los estudiantes y profesores que participaron en esta investigación compartiendo su tiempo y experiencias. Gracias por su confianza y su aportación a la investigación educativa.

**, *** Instituto de Investigación y Desarrollo Educativo-Universidad Autónoma de Baja California. Correo electrónico: claudia.salinas.boldo@uabc.edu.mx

**** Facultad de Idiomas Ensenada-Universidad Autónoma de Baja California.
}

Recibido el 04 de octubre del 2017; aceptado el 22 de septiembre del 2018.

\section{Resumen}

El presente trabajo tuvo el objetivo de analizar la forma en la que se construyen las literacidades académico-investigativas en licenciaturas del área de las ciencias sociales, en tres universidades públicas del norte, centro y sur de México. Como técnicas de investigación de una metodología de orientación cualitativa, se aplicaron observaciones, entrevistas, grupos focales y recolección de documentos.

Los resultados indican que si bien los estudiantes logran desarrollar algunas habilidades investigativas, no están participando de las prácticas y los significados de los investigadores de su disciplina, lo cual obstaculiza la construcción de una identidad como investigadores o futuros investigadores.

Palabras clave: Literacidad académica, literacidad investigativa, literacidad científica, educación superior, universidad. 


\begin{abstract}
This research had the purpose of analyzing the way in which investigative-academic literacies are constructed in university programs of the area of the social sciences, in three public universities of the north, center and south of Mexico. As research techniques of a qualitative orientation methodology, the observations, interviews, focus groups and documentary collection were applied.

The results indicate that despite students are able to develop some investigative skills, they are not participating in the practices and meanings of the researchers of their discipline, which hinders the construction of an identity as researchers or future researchers.
\end{abstract}

Keywords: Academic literacy, Investigative literacy, scientific literacy, Higher education, University.

\title{
Introducción
}

$\mathrm{D}$ entro del mapa o malla curricular de las licenciaturas ubicadas dentro del área de las ciencias sociales, encontramos asignaturas orientadas al desarrollo de habilidades investigativas. Estas asignaturas conforman el área de investigación o de metodología. Cabe mencionar que nos estamos refiriendo a una situación común a programas impartidos en universidades públicas, específicamente aquellas involucradas en este estudio.

Las asignaturas del área de investigación o de metodología son asignaturas enfocadas en la formación de los estudiantes como investigadores en su campo o disciplina. Se cursan desde las primeras etapas de la licenciatura hasta el final, con el objetivo de que el futuro profesionista cuente con un entrenamiento, al menos básico, en materia de investigación. En la universidad se espera que los estudiantes desarrollen habilidades y adquieran conocimientos que les permitan llevar a cabo proyectos de investigación. Se considera que el estudiante, al final de su proceso formativo, debe ser capaz de comprender y producir textos académicos, lo cual implica un cierto nivel de conocimiento en torno a las formas de producción de conocimiento en su campo o disciplina. Sin embargo, estas expectativas no siempre se cumplen. (Rojas-Betancur y Méndez-Villamizar, 2013).

El presente trabajo es el resultado de una investigación llevada a cabo durante doce meses, de agosto de 2016 a agosto de 2017, en tres universidades públicas del país. El objetivo de esa investigación fue el de conocer las prácticas de lectura y escritura que se dan en las clases de metodología, de varias 
licenciaturas del área de las ciencias sociales, y analizar la forma en la que esas prácticas constituyen literacidades académico-investigativas y aportan a la construcción de una identidad en los estudiantes como investigadores en sus disciplinas.

Convertir al estudiante universitario en investigador no se trata de brindarle un adorno adicional ni es un lujo superfluo (Soria, 2005). Entrenar en investigación implica la construcción de sujetos epistémicos. Se trata, entre otras cosas, de reflexionar en torno a las formas de generación de conocimiento que son propias de cada campo o disciplina. En el proceso de enseñar a investigar, tendríamos que promover el desarrollo de habilidades en torno al planteamiento de preguntas y no a la memorización de respuestas ya construidas de antemano. El estudiante tiene que adquirir conocimientos y desarrollar habilidades, pero también actitudes - y valores - que acompañan a la práctica investigativa (Rizo, 2006). Es importante trascender la mera transmisión de conocimientos o de una técnica, para llegar a la transmisión de una cultura investigativa (Rojas-Betancur y Méndez-Villamizar, 2013).

Una problemática común que se presenta en asignaturas del área de metodología, es la baja motivación de los estudiantes, quienes suelen presentar actitudes de resistencia o rechazo hacia estas asignaturas (Morales; Rincón y Tona, 2005). Los estudiantes de metodología preguntan a sus profesores: "¿Y esto para qué me va a servir?", y los profesores no siempre tienen la respuesta (Acevedo, 2004).

Otro problema tiene que ver con la lectura y la escritura. Los profesores universitarios en general consideran que los estudiantes no leen ni escriben de manera adecuada y suelen echar la culpa de esta situación a etapas educativas anteriores (Carlino, 2003)

Sánchez et al. (2013) nos dicen que la escritura es la "tecnología privilegiada" a través de la cual se construye y se transmite el conocimiento. Es el requisito indispensable para acceder a las comunidades disciplinares e institucionales. Además, la escritura es una forma de encontrar, construir y compartir significados de una forma colectiva (Ramos, 2009). Entonces, es necesario entender la forma en la que se están llevando a cabo las prácticas de lectura y escritura en los contextos educativos, para por un lado poder abordar problemáticas relacionadas con la motivación y la escritura de los estudiantes, y por otro, entender las formas en las que, como docentes y autoridades, podemos promover aprendizajes más completos y significativos en nuestros estudiantes. Más allá de esto, resulta oportuno el entender estas prácticas de lectura y escritura como prácticas de literacidad, pues esto nos permite entender a la lectura y la escritura como hechos profundamente vinculados con lo social, constructores de comunidades, de significados, de 
identidad y de esas culturas disciplinares que tendríamos que transmitir en la universidad, a la par de los conocimientos y las habilidades técnicas.

\section{Literacidades investigativas ¿Cómo?}

Entendemos la literacidad o literacidades como prácticas sociales en las cuales la lectura y la escritura son acciones protagonistas, siempre orientadas hacia una meta vinculada con lo social. Estas prácticas siempre estarán atravesadas por la cultura, la historia, los significados y las identidades de los lectores y escritores. Es decir que el leer y el escribir son acciones que no pueden entenderse como independientes de factores históricos, contextuales y subjetivos. Las literacidades son afectadas por estos factores y, a su vez, contribuyen a moldearlos (Gee, 2015).

Wenger (2010) indica que el aprendizaje no es un proceso individual que se reduzca a la mera acumulación de habilidades y conocimientos. El aprendizaje ocurre en colectividad e involucra la construcción de significados e identidades. El estudiante es un sujeto activo, que construye junto con otros y se transforma mientras aprende.

Las prácticas de literacidad son siempre sociales, las formas en las que se usa el lenguaje escrito en una cultura determinada, y existen diferentes literacidades vinculadas con distintos dominios de la vida (Barton y Hamilton, 2000). Para entender la forma en la que se dan los aprendizajes, tendríamos que entender las prácticas de lectura y escritura que hacen posible que ocurran estos aprendizajes, en su contexto social, cultural e histórico.

Las literacidades investigativas son aquellas que se producen con el objetivo de construir conocimiento dentro de un campo o disciplina, siguiendo un método y ciertos lineamientos, consensualmente establecidos por una comunidad determinada de especialistas. Estas comunidades de práctica construyen y negocian significados en colectivo. Los participantes de estas comunidades de práctica se encuentran siempre activos, son creadores y consumidores permanentes de significados. El proceso de aprender involucra la apropiación de ciertos conocimientos, el desarrollo de habilidades y la incorporación de ciertos valores, pero también implica la construcción de una cierta identidad (Wenger, 2010). En el caso del tema que nos ocupa, estaríamos hablando de la construcción de una identidad como profesionista investigador, heredero de saberes, de historia y de significados, que son producto de un camino recorrido por la comunidad de práctica a la que aspira pertenecer.

Cuando los estudiantes universitarios cursan asignaturas del área de metodología, se enfrentan a la tarea de construir un proyecto de investigación, 
y esto puede resumirse, básicamente, en la realización de dos actividades: lectura de textos académicos y escritura de al menos un texto académico propio. La construcción de un texto académico propio, aunque sea de forma incipiente, es una actividad considerada como favorable al aprendizaje por diversos autores (Morales, Rincón y Tona, 2005; Perdomo, 2007; Pozuelos y Travé, 2004; Rizo, 2006; Sánchez, 2014; Sancho, 2001 y Trejo y García, 2006). quienes coinciden en señalar que es necesario que los estudiantes desarrollen una investigación para poder aprender a investigar.

Los profesores de ciencias sociales y humanidades tenemos ciertas expectativas con respecto a las prácticas de lectura y escritura de nuestros estudiantes. Pero tendríamos que considerar, por un lado, que el texto académico es un tipo de texto nuevo y desconocido para el estudiante universitario y, por otro, que la lectura que nosotros esperamos que él haga de éste sería propia de una "cultura lectora disciplinar", es decir, característica de esa disciplina en particular, que responde a ciertas necesidades y significados relacionados con la forma de construir y consumir el conocimiento en esa disciplina en particular. Al estudiante entonces le faltan códigos para entender unos textos que no van dirigidos a ellos como estudiantes sino a los profesionales, a los académicos. Nosotros, como profesores, tendríamos que no solamente transmitirles esos códigos sino darles la bienvenida a esta nueva cultura de la que empiezan a formar parte (Carlino, 2003 b). A propósito de lo anterior, rescatamos lo dicho por Brown (1989), quien nos habla de la enculturación como algo que ocurre cuando un estudiante, a fuerza de involucrarse en actividades prácticas, aprende a leer y escribir como lo hacen los especialistas, pues no se trata simplemente de reproducir técnicas y acumular conocimientos, sino de involucrarse en esas mismas actividades que llevan a cabo los profesionales de la disciplina.

Una de las habilidades que un estudiante de metodología requiere para construir textos académicos es la de la argumentación (Campanario, 2004; Sánchez et al., 2013). Castro y Sánchez (2013) llevaron a cabo el análisis de 40 ensayos de opinión de estudiantes de la licenciatura en lenguas modernas de una universidad pública mexicana, y encontraron que los estudiantes presentan dificultades para construir textos académicos argumentativos. Citan a los autores o hacen alguna referencia a ellos, pero esto termina siendo un "recurso de incorporación de saberes validados", es decir que los estudiantes consideran lo dicho por los autores como descripciones de una verdad que no se cuestiona. Se adhieren a lo enunciado por el experto para validar su opinión, es decir, reproducen voces ajenas en vez de construir una propia. Castro y Sánchez resaltan la importancia de desarrollar competencias argumentativas en los estudiantes para generar un pensamiento más complejo y 
reflexivo, y para que éstos “[...] se reconozcan como sujetos con voz, capaces de opinar y defender posturas [...]", algo que es fundamental cuando hablamos no solamente de hacer investigación y redactar textos académicos, sino de compartirlos en espacios propios de la comunidad disciplinar.

Existen ciertas prácticas y estrategias identificadas como efectivas cuando se trata de favorecer el desarrollo de habilidades investigativas en los estudiantes. Estas prácticas son: contar con profesores que sean, ellos mismos, científicos (Bozu y Canto, 2009; Méndez-Villamizar, 2013; Rojas-Betancur y Sánchez, 2014); lectura crítica de artículos científicos (Britt, 1995; Campanario, 2004; Morales, Rincón y Tona, 2005); acompañamiento al profesor en alguna investigación que éste se encuentre realizando (Morales y Romero, 2005); el transmitir no solamente una técnica sino una cultura de la investigación (Pozuelos y Travé, 2004); que los profesores se formen en torno a los avances científicos que se estén dando en su área de especialidad (Gil y Pessoa, 2000); ligar los temas de la clase con fenómenos de la vida cotidiana, de tal manera que los alumnos le encuentren sentido a aquello que aprenden en el salón de clases y el aprendizaje sea significativo (Pedrinaci , 2012 y Pelcastre, Gómez y Zavala, 2015); acompañamiento del estudiante en todas las partes del proceso (Perdomo, 2007) y publicación de artículos y presentación en congresos (Soto, 2011).

Morales, Rincón y Tona (2005) sugieren el abordaje de problemáticas sociales reales en el salón de clases, acercar a los estudiantes a la lectura de situaciones actuales desde los textos académicos que se producen en la disciplina en la que se están formando. Adicional a la lectura de textos académicos, los autores sugieren a los profesores pedir a los estudiantes que construyan sus propios proyectos de investigación; acompañar al estudiante en cada fase de estos proyectos; que estos proyectos se lleven a cabo en $-\mathrm{y}$ también, sería deseable, con - la comunidad; que los proyectos sean expuestos en clase por los estudiantes-autores; compartir sus propias experiencias como investigador; mantener una relación asertiva con los estudiantes y practicar la "investigación significativa", es decir, llevar a cabo una práctica investigativa que motive a los estudiantes y les deje clara la importancia que la investigación tiene para el avance de su disciplina y de la sociedad en general.

Las recomendaciones de los especialistas podrían resumirse en lo dicho por Perdomo (2007), quien nos dice que es un error enseñar a investigar a partir de resultados de la investigación. Es decir, no basta con leer investigaciones y manuales de metodología de la investigación. A investigar se aprende investigando y contando con la compañía del profesor en cada una de las partes del proceso. 
Una de las situaciones que favorecen la falta de motivación de los estudiantes hacia el quehacer investigativo es el desconocimiento que existe en torno a lo que hacen los investigadores. Pelcastre, Gómez y Zavala (2015), quienes aplicaron un protocolo de actitudes hacia la ciencia en estudiantes mexicanos de media superior, encontraron que los estudiantes consideran que los investigadores gozan de una buena aceptación social, pero que la ciencia no tiene aplicaciones prácticas para resolver problemas reales. También se encontró que los estudiantes desconocen lo que hacen los investigadores y la relevancia que tiene su labor. Los autores recomiendan vincular los temas de la clase con la vida cotidiana para comprender las aplicaciones prácticas que tiene la ciencia y construir una relación de empatía con los estudiantes. Cabe mencionar que aunque el protocolo utilizado por Pelcastre, Gómez y Zavala hacía referencia a las ciencias exactas y naturales, consideramos que el caso de las actitudes de estudiantes hacia las ciencias sociales no es demasiado diferente.

\section{Literacidades investigativas. ¿Para qué?}

El compromiso de la universidad pública va más allá de la simple capacitación de futuros profesionistas para la realización de un trabajo especializado. Se espera que la universidad promueva en sus estudiantes el interés por el cambio social y el bienestar común (Acevedo, 2004; Rivera, 2014; Rizo, 2006; Rodríguez et al., 2010 y Sánchez, 1990.) Rivera (2014), además, nos dice que la escuela es un espacio en el que se construye ciudadanía. Más allá de las calificaciones y la capacitación para el empleo, la autora indica que la escuela tendría que ser el lugar en el que se adquieren herramientas para participar en la vida social y laboral de una forma más constructiva.

Acevedo (2004) se pronuncia a favor de una "educación científica" que promueva en los estudiantes tanto conocimientos como habilidades, actitudes y valores útiles a un ejercicio más crítico y consciente de la ciudadanía. Los ciudadanos, a decir del autor, tienen la posibilidad de tomar decisiones que afecten la vida pública, y es mejor contar con una ciudadanía no solamente más informada sino formada en el quehacer investigativo, de tal manera que pueda comprender de manera más amplia y compleja los fenómenos de la vida cotidiana. Por su parte, Pedrinaci (2012) nos habla de la necesidad de desarrollar una "competencia científica" en los estudiantes universitarios. Esta competencia científica comprendería un conjunto de capacidades relacionadas con la aplicación del método científico para: 
- Describir, explicar y predecir fenómenos cotidianos.

- Analizar problemáticas y tomar decisiones.

- Experimentar la curiosidad científica, buscar y seleccionar información relevante.

- Construir un argumento.

- Analizar conclusiones a la luz de hechos, datos, observaciones y experiencias empíricas.

- Comprender la diferencia entre lo científico y lo no científico.

- Entender cómo se construye el conocimiento.

- Valorar la influencia social de los productos de la ciencia y debatir sobre ello.

- Asumir responsabilidades como ciudadano y profesionista, y desarrollar competencias lingüísticas que le permitan leer, escribir y hablar en el código de los científicos de su campo o disciplina.

Si bien el autor, cuando nos habla de ciencia, lo hace haciendo alusión a las ciencias naturales y exactas, sus argumentos son igualmente oportunos para el caso de las ciencias sociales. Rodríguez et al. (2010) se pronuncian a favor de la formación en investigación, no solamente para promover la vocación científica, sino para formar profesionales comprometidos con las diversas problemáticas sociales. Asimismo, indica que es necesario pensar en la ciencia no como algo estático y acumulativo sino como un proceso, una construcción social e histórica dinámica y siempre influenciada por factores políticos, culturales e ideológicos.

Morales y Cassany (2008) nos dicen que “[...] cada disciplina constituye una comunidad discursiva, que se conforma a partir del uso de unas prácticas lectoras y escritoras particulares [...]". Estas prácticas se articulan en torno a un campo o área del conocimiento, a determinadas prácticas sociales y a ciertos géneros discursivos. Es en la universidad que los estudiantes - futuros miembros de una disciplina - conocen los géneros propios de cada disciplina, así como las formas de lectura y escritura que éstos requieren. El aprendiz entonces es entrenado en estos saberes que constituyen la literacidad especializada, que lo habilitarán para convertirse en un miembro activo de una comunidad disciplinar. Los autores indican que la lectura y la escritura son actividades profundamente vinculadas con las formas de transmitir y hacer uso del conocimiento al interior de las disciplinas. Leer y escribir, como erróneamente pensamos a veces los profesores, no son habilidades que los estudiantes ya deberían de traer o habilidades que son independientes del entrenamiento en un campo del saber. La literacidad, como práctica social mediada por textos, se va desarrollando a la par de la adquisición de conocimientos y habilidades específicos de una profesión, y eso es algo que, idealmente, tendría que ocurrir en la universidad. 
La universidad tiene el compromiso de promover ciertas habilidades en sus estudiantes, tales como la creatividad, el pensamiento crítico, la disciplina y la objetividad, entre otras. El entrenamiento en investigación es una forma eficaz de promover la adquisición de estas habilidades en los estudiantes, así como de motivarlos a construir posturas y argumentos tanto pertinentes como bien fundamentados ante los temas afines a su disciplina. El entrenamiento en investigación es una forma de adquirir el conocimiento de la disciplina, no algo que se deba de aprender posteriormente como habilidad adicional. La investigación tendría que ser vista como un medio eficaz para acceder a los conocimientos y las prácticas características de cada profesión (Soria, 2015).

\section{Procedimiento}

Este estudio se llevó a cabo desde la metodología cualitativa y se aplicaron las siguientes técnicas: observación, entrevistas individuales a estudiantes, entrevistas grupales y grupos focales. Esto queda plasmado en la siguiente tabla, en la que los números fuera de paréntesis indican el número de veces que la técnica se aplicó en cada licenciatura, y los números dentro de los paréntesis, en el caso de la entrevista grupal y el grupo focal, indican el número de participantes.

Tabla 1.

Técnicas y participantes

\begin{tabular}{|c|c|c|c|c|c|}
\hline & \multicolumn{2}{|c|}{ Universidad del Norte } & \multicolumn{2}{|c|}{ Universidad del Centro } & \multirow{2}{*}{$\begin{array}{c}\begin{array}{c}\text { Universidad } \\
\text { del Sur }\end{array} \\
\text { Educación }\end{array}$} \\
\hline & $\begin{array}{c}\text { Ciencias de la } \\
\text { Educación }\end{array}$ & Psicología & Pedagogía & $\begin{array}{l}\text { Sociología } \\
\text { Educativa }\end{array}$ & \\
\hline Observación & & 1 & 2 & 2 & 1 \\
\hline $\begin{array}{l}\text { Entrevista } \\
\text { individual a } \\
\text { estudiantes }\end{array}$ & 2 & 1 & 2 & 1 & 2 \\
\hline Entrevista grupal & & $1(17)$ & $1(19)$ & & \\
\hline Grupo focal & & $1(3)$ & & $1(4)$ & $1(4)$ \\
\hline
\end{tabular}

En todas las licenciaturas se llevó a cabo la recolección de los mapas curriculares y los planes de estudio. También se recolectaron imágenes de apuntes de los cuadernos de los estudiantes y presentaciones de proyectos.

Para efectos del presente escrito, se identifican las universidades participantes en esta investigación como: Universidad del Norte, Universidad del 
Centro y Universidad del Sur. Además, se omiten los nombres de los y las estudiantes que participaron en este estudio para proteger su identidad.

El trabajo de campo se llevó a cabo en asignaturas del área de metodología de la investigación ubicadas dentro de la etapa disciplinar de las licenciaturas. Los estudiantes que participaron en este estudio se encontraban cursando la segunda mitad de sus licenciaturas.

El acceso a los espacios de campo, en psicología, pedagogía, sociología educativa y educación, se logró con el apoyo de los profesores, quienes accedieron a dejarnos observar sus clases y nos presentaron a sus estudiantes. En el caso de ciencias de la educación, el contacto se hizo directamente con los estudiantes. La participación en las entrevistas y los grupos focales por parte de los estudiantes fue voluntaria, y se llevó a cabo en las instalaciones universitarias. Con respecto a las entrevistas grupales, se realizaron en el espacio de clase de los profesores y se aclaró a los estudiantes que no estaban obligados a participar ni a responder a todas las preguntas, a menos que así lo desearan. En la entrevista grupal que se llevó a cabo con el grupo de psicología de la Universidad del Norte, aproximadamente la mitad del grupo participó, mientras que en la entrevista grupal que se aplicó en el grupo de Pedagogía de la Universidad del Centro, todos los estudiantes participaron.

Tanto las entrevistas como los grupos focales fueron audiograbados, a excepción de una parte de una entrevista individual que no fue registrada en audio pero sí en notas de campo, atendiendo a una petición de la persona entrevistada. Dicha petición ocurrió porque durante esa parte de la entrevista, la estudiante expresó disconformidad con la forma de trabajar y las actitudes de uno de sus docentes y temía posibles represalias.

Durante las entrevistas individuales y los grupos focales se les solicitó a los estudiantes que nos hablaran acerca de su experiencia en las asignaturas del área de metodología de la investigación, específicamente de los siguientes temas:

- Textos utilizados como bibliografía básica (el libro o libros marcados como obligatorios por el profesor).

- Textos complementarios (aquellos a los que tuvieron que recurrir para construir su proyecto de investigación).

- Estrategias de búsqueda de información, tanto las aprendidas en clase como aquellas desarrolladas por su cuenta.

- Uso de los textos académicos.

- Estrategias de escritura, tanto las adquiridas en clase como aquellas desarrolladas por cuenta propia o adquiridas en otros espacios.

- Participación en eventos académicos: concepción de la investigación dentro de su disciplina. 
- Actitudes hacia la investigación y el quehacer investigativo y calidad de la relación con el docente de la asignatura.

En esta investigación se utilizaron consentimientos informados. Cabe mencionar que nuestros consentimientos informados constan de dos documentos: uno, firmado por el participante, que conservamos nosotros como autores, y otro, firmado por la investigadora titular que se le da al participante. De esta manera a nosotros se nos autoriza hacer uso de la información proporcionada y existe un compromiso hacia los participantes de hacer uso de esa información para fines exclusivamente académicos.

\section{Resultados}

Los datos obtenidos durante la etapa de trabajo de campo se organizaron en tres categorías deductivas: identificación, significados y estrategias.

\section{1.- Identificación}

La palabra identificación en este apartado se toma en un doble sentido: el de la identificación como ubicación y descripción de aquellos elementos que forman parte de un conjunto y el de la identificación como ese proceso que se pone en marcha cuando nos reconocemos en algo o alguien más.

1.1.- Tomando a la identificación, en el sentido de aquello que se ubica en un contexto y se describe, se encontró que los estudiantes no identifican muchos elementos que son parte de la "vida real", concreta y práctica de los investigadores. Los estudiantes no saben en dónde se encuentran los investigadores de su disciplina, sobre qué temas escriben, la forma en la que llevan a cabo su trabajo, los espacios en los que se desenvuelven, cuánto ganan y qué tuvieron que hacer para llegar ahí. Muchos de ellos no saben cuáles son los recursos con los que cuentan como estudiantes de licenciatura, ni el camino a seguir para convertirse en investigadores. Durante las entrevistas, ocurrió que una estudiante manifestó sorpresa al enterarse de que eso que nosotros hacíamos - trabajo de campo para una investigación - fuera un trabajo remunerado. Otro estudiante dijo que le gustaba la investigación pero que pensaba dejarla para sus "ratos libres", es decir, no adoptarla como actividad formal y principal fuente de ingresos, porque consideraba que la investigación no era un trabajo que generara remuneración económica suficiente para subsistir, además de que - para llegar a ser investigador - se requiere tener estudios 
de posgrado y eso "[...] cuesta mucho dinero y, mientras tanto [mientras se cursan los estudios de posgrado], ¿Uno de qué vive?".

1.2.- Pensando en la identificación, como proceso a través del cual nos reconocemos en algo o alguien más, concluimos que los alumnos observados y entrevistados en general no se identifican con el quehacer investigativo ni con los investigadores. Cuando cuestionábamos a los estudiantes acerca de la trayectoria profesional de sus maestros, todos parecían estar muy orgullosos del hecho de que sus profesores - todos - eran investigadores además de docentes. Nos dijeron que sus profesores publican libros y artículos, que acuden a congresos y de algunos de ellos nos dijeron que son parte del SNI (Sistema Nacional de Investigadores). A un par de estudiantes les preguntamos ¿Qué es el SNI? y no pudieron responder. También preguntamos acerca de las líneas de investigación y las publicaciones de sus profesores, y los estudiantes tampoco pudieron responder. Nos encontramos con que los profesores no suelen compartir información acerca de su trabajo con los alumnos.

Es probable que lo anterior sea uno de los factores causantes de que los universitarios no tomen a sus profesores de metodología de la investigación como modelos a seguir, independientemente de la calidad de relación profesor-alumno que ellos perciben. Otra de las razones que pudiera estar detrás de esta no-identificación con el investigador y su labor, está relacionada con la baja participación de los estudiantes como ponentes en eventos académicos. Los alumnos son informados en clase o por medio de carteles, de eventos tales como congresos y coloquios. Se les invita a asistir y se menciona la posibilidad de que ellos como estudiantes presenten algún trabajo, pero esto último es algo que los alumnos, en general, no consideran seriamente.

En todas las universidades visitadas se han dado casos - pocos - de estudiantes que se presentan como ponentes en un congreso, y esto siempre ha sido resultado de una invitación directa por parte del profesor, quien anima y asesora al estudiante a presentarse con algún trabajo, previamente elaborado para aprobar su materia. En uno de estos casos el profesor se presentó, a la par de sus estudiantes, como ponente en el congreso, e incluso presentó su propia investigación en la misma mesa. Los estudiantes que han participado como ponentes en estos eventos, siempre terminan considerando la investigación como opción de trabajo a futuro, y considerándose a sí mismos como investigadores, especialistas en ese trabajo específico que han presentado. Pero es necesario recordar que estos casos son la excepción, pues la participación como ponentes en eventos académicos no es una experiencia común entre los estudiantes. 


\section{2.- Significados}

Hablando de las calificaciones en las materias de metodología de la investigación, la tendencia general de los estudiantes es a aprobar. Sin embargo, la investigación es algo que, salvo algunas contadas excepciones, no disfrutan hacer. Desde el momento que planteábamos el tema a los participantes, éstos expresaban disgusto e incomodidad, no ante el hecho de ser interrogados, sino ante el tema en particular. Consideran que la investigación es "aburrida", "difícil" y "sin sentido". El tema de la dificultad percibida es algo que abordaremos en el planteamiento de la categoría de estrategias. Ahora vincularemos el aburrimiento con la falta de sentido, para ahondar en el tema de los significados. Algo que, sin excepción, fue común a todos los estudiantes que participaron en este estudio, es la creencia de que la investigación debe de "servir para algo". Los estudiantes están convencidos de que la investigación cobra sentido cuando es útil para resolver alguna problemática social, lo cual creemos tal vez se deba al perfil de las licenciaturas orientadas al trabajo con y para las personas. Una labor que no es percibida como dotada de sentido, es una labor que termina por aburrir a quien la realiza.

Sin necesidad de plantear ninguna pregunta al respecto, los estudiantes se adelantaron a dar sugerencias para solucionar la falta de sentido de la cual se quejaron. Ellos ven como un problema el hecho de tener que iniciar un nuevo proyecto cada semestre - uno diferente para cada materia del área de investigación - y el hecho de tener que hacer dos proyectos distintos en un mismo semestre, para materias que podrían ser complementarias. Sugieren que los profesores "se pongan de acuerdo" y pidan un solo proyecto, con objetivos para ambas materias - en el caso de materias de un mismo semestre que pudieran complementarse - y que se lleve un solo proyecto de investigación a lo largo de la carrera, que vaya creciendo y enriqueciéndose a medida que se avanza en las asignaturas. Asimismo, consideran que ese proyecto de investigación tendría que incluir, invariablemente, una fase final en la cual se realice una intervención que beneficie a alguien concreto.

Algo que es necesario aclarar, es que algunos profesores - todos, en el caso de pedagogía, de la Universidad del Centro - piden a los estudiantes que sus proyectos de investigación cuenten con una etapa de intervención, o piden proyectos de intervención "fundamentados", es decir, la descripción de un taller de varias sesiones, con un marco teórico a manera de introducción, lo cual nos parece que genera una confusión en los estudiantes, quienes terminan considerando que la investigación y la intervención son la misma cosa.

Lo que consideramos importante conservar, como una práctica observada en todos los escenarios de campo, es el permitir que los estudiantes sean los que elijan el tema de su proyecto. Con respecto a este punto, encontramos 
una sola excepción, una profesora que tenía una breve lista de temas "prohibidos" pues argumentaba que esos eran temas populares - entre estudiantes y académicos - y por lo tanto "ya muy estudiados", por lo cual les pedía a sus estudiantes que propusieran temas distintos y para esto les sugería que leyeran y observaran a su alrededor.

El hecho de poder elegir el tema de su proyecto es algo que identificamos como uno de los principales elementos motivadores hacia el trabajo investigativo, ya que los alumnos dicen estar interesados en resolver inquietudes propias que han surgido en sus vidas personales, sus contextos laborales o algo que consideran como un tema o problemática urgente a tratar en su contexto inmediato.

\section{3.- Estrategias}

Para iniciar la exposición de esta tercera categoría, retomaré la queja de los estudiantes en torno a lo "difícil" que les resulta la investigación. Ahondando en torno a esta afirmación, nos encontramos con otro hallazgo importante que puede sintetizarse en el siguiente comentario que hizo una estudiante, refiriéndose a los proyectos de investigación que los profesores les piden: "Nos dicen qué, pero no nos dicen cómo". Con esto quería decir que los profesores les indican las partes que debe de tener el proyecto de investigación, el orden en el que deben de ir y les brindan descripciones generales de éstas, pero no les dan estrategias para llevar a cabo: a) La lectura de textos académicos y b) La escritura del proyecto, ambas actividades fundamentales para lograr una investigación.

Para la búsqueda de textos académicos, cuentan con talleres de dos a cuatro horas - en promedio - impartidos por personal de la biblioteca, el que los introduce al tema del uso de las bases de datos. Los estudiantes conocen los sitios en los cuales pueden conseguir literatura académica, aunque desconocen las razones que hacen que un texto académico sea distinto de uno de divulgación popular. En una sesión de entrevista grupal, se planteó a los estudiantes la siguiente pregunta: "¿qué diferencia hay entre un texto académico y uno de divulgación popular?", a lo que ellos respondieron que el texto académico es aquel que se encuentra en ciertas bases de datos, mientras que el texto de divulgación popular es el que se consigue en los puestos de periódicos o aquellos que se encuentran en páginas no reconocidas - o incluso, abiertamente rechazadas - por la comunidad académica. Cabe destacar que esta respuesta vino de parte de algunos estudiantes, mientras que otros, mirándonos con extrañeza, nos pidieron que respondiéramos a la pregunta que acabábamos de plantear. 
Con respecto a la lectura, los profesores les dan formatos para elaborar fichas de trabajo. Algunos de esos formatos se encuentran en manuales de metodología, y otros son que los profesores proponen, sin que medie ninguna referencia a textos o autores. En contadas ocasiones nos encontramos con fichas de trabajo que eran idénticas a las que el profesor proponía. En los estudiantes hay una tendencia general a personalizar estos formatos, agregando o eliminando elementos con base en sus necesidades de escritura, es decir, pensando en tomar y acomodar la información en la ficha de trabajo, de tal manera que la citación, el parafraseo y en general la construcción del proyecto se hicieran más sencillos.

Un problema común que se presenta con la lectura de textos académicos es el encuentro con vocabulario nuevo, que dificulta la lectura de los documentos. Una estrategia común es la de utilizar diccionarios. Una estudiante, además, escribía estas nuevas palabras con su significado en papeles autoadheribles de colores, con los cuales cubrió un cuadro que se encontraba en la parte de la casa en la que acostumbraba sentarse a trabajar. Según nos explicó, ella consideraba si estaba leyendo textos que giraban en torno al mismo tema de investigación, entonces era muy probable que volviera a toparse con esas mismas palabras, y para no regresar al diccionario una y otra vez, ella prefería copiarlas y tenerlas a la mano mientras trabajaba. Cabe mencionar que hay estudiantes que en vez de desarrollar estrategias para enfrentar la aparición de palabras nuevas, prefieren buscar textos "más fáciles", es decir, con un vocabulario percibido como más accesible.

En los estudiantes existe una tendencia importante a hacer uso de dispositivos electrónicos y aplicaciones para leer. Esto es particularmente marcado en los estudiantes de la Universidad del Centro, dado que pasan muchas horas en el transporte público, lo cual los lleva a aprovechar estos tiempos de traslado para adelantar la lectura de textos.

Al momento de escribir, los estudiantes parecen toparse con más problemas y contar con menos estrategias. Existe una tendencia general a hacer notas en los dispositivos electrónicos, cuadernos, libros, servilletas y notas de compra, para después utilizarlos como insumo para escribir el proyecto de investigación.

Los estudiantes confunden la argumentación con "dar mi opinión"; no están seguros de "lo que tiene que ir" en cada parte de la investigación - aunque enlistan de memoria las partes de una investigación - y abusan de la cita textual, porque el parafraseo se les dificulta. Como ellos mismos dicen, no basta con saber el qué, necesitan que sus profesores les muestren el cómo. 


\section{Conclusiones}

Nos parece importante iniciar este apartado destacando aquello que ya se está llevando a cabo, tanto por las universidades como por los profesores y los estudiantes, en materia de desarrollo de literacidades investigativas. Las universidades brindan información a los estudiantes acerca de eventos académicos y destinan recursos para que asistan como ponentes. Asimismo, organizan eventos tales como conferencias y foros con la intención de dar a conocer el trabajo de los investigadores. Los profesores, por su parte, los motivan a construir un proyecto de investigación que gire en torno temas de su interés, y animan a los estudiantes a participar en los eventos, funcionando como asesores para aquellos estudiantes que deciden convertirse en ponentes.

Ante las dificultades que la escritura y la lectura de textos académicos les plantean, los estudiantes han desarrollado estrategias tales como la elaboración de glosarios y notas, uso de dispositivos y personalización de formatos de fichas de trabajo, para llevar a cabo la construcción de su proyecto.

De acuerdo con los estudiantes, los profesores les dejan claro el qué -la estructura - de la investigación, pero no les hablan del cómo, es decir, no comparten con ellos estrategias de lectura y escritura. De la misma forma, los profesores - que son a su vez investigadores - no comparten con ellos la cultura del mundo de la investigación, es decir, esas prácticas sociales, significados, códigos, formas e incluso el camino a seguir para convertirse en un investigador, lo cual ocasiona que los estudiantes no comprendan el sentido e incluso la utilidad de esta labor y los productos que ésta genera. Los alumnos se preguntan: "¿Y esto para qué me va a servir?”, pues consideran que la investigación en sus disciplinas - todas del área de las ciencias sociales - deben de ser útiles para resolver alguna problemática de su contexto inmediato, pues como mera acumulación de conocimiento teórico, el quehacer investigativo les parece una labor sin sentido.

Los estudiantes logran encontrar un sentido a la investigación, e incluso verse como investigadores, cuando consiguen entender e incluso participar de esas prácticas sociales que convierten la acción de leer y escribir textos académicos en prácticas de literacidad investigativas, propias de una cultura disciplinar compleja y rica en significados.

\section{Referencias}

Acevedo, José Antonio (2004). Reflexiones sobre las finalidades de la enseñanza de las ciencias: educación científica para la ciudadanía. Revista Eureka sobre En- 
señanza y Divulgación de las Ciencias, 1 (1), 3-15. Recuperado de: http:/ / rodin. uca.es/xmlui/bitstream/handle/10498/16530/Reflexiones \%20sobre $\% 20$ las $\% 20$ finalidades $\% 20 \mathrm{de} \% 201 \mathrm{la} \% 20$ ense $\%$ C3\%B1anza $\% 20 \mathrm{de} \% 201$ as $\% 20$ ciencias.pdf?sequence $=1$ \&isAllowed $=y$

Bozu, Zoia y Pedro José Canto (2009). El profesorado universitario en la sociedad del conocimiento: competencias profesionales docentes. Revista de Formación e Innovación Educativa Universitaria, 2 (2), 87-97. Recuperado de: http:/ / padula. detodoproducciones.com.ve/IS3_1_El\%20profesorado\%20universitario.pdf

Britt, Michael A. (1995). Research on Trial: A Pedagogy for Research Methods Instruction. Recuperado de: http:/ / files.eric.ed.gov/fulltext/ED389374.pdf

Campanario, Juan Miguel (2004). Algunas posibilidades del artículo de investigación como recurso didáctico orientado a cuestionar ideas inadecuadas sobre la ciencia. Enseñanza de las Ciencias, 22 (3), 365-378. Recuperado de: http://ddd.uab.cat/pub/edlc/02124521v22n3/02124521v22n3p365.pdf

Cassany, Daniel y Oscar Morales (2008). Leer y escribir en la universidad: Hacia la lectura y la escritura crítica de géneros científicos. Revista Memoralia, 5 (2), 69-82.

Gee, James Paul (2015). The new literacy studies. En: Rowsell, Jennifer y Kate Pahl (Eds.) The Routledge Handbook of Literacy Studies. USA: Routledge

Gil, Daniel y Anna María Pessoa (2000). Dificultades para la incorporación a la enseñanza de los hallazgos de la investigación e innovación en didáctica de las ciencias. Educación Química, 11 (2), 250-257.

Morales, Oscar Alberto; Rincón, Ángel Gabriel y José Tona (2005). Cómo enseñar a investigar en la universidad. Universidad de los Andes. Recuperado de: http:/ / www.saber.ula.ve/bitstream/123456789/19967/2/articulo9.pdf

Morales, Oscar Alberto y Daniel Cassany (2008). Leer y escribir en la universidad: Hacia la lectura y la escritura crítica de géneros científicos. Revista Memoralia, 5 (2), 69-82.

Pelcastre, Leticia; Gómez, Alma Rosa y Genaro Zavala (2015). Actitudes hacia la ciencia de estudiantes de educación preuniversitaria del centro de México. Revista Eureka sobre Enseñanza y Divulgación de las Ciencias, 12 (3), 475-490. Recuperado de: http://reuredc.uca.es/index.php/tavira/article/viewFile/821/pdf_319

Pedrinaci, Emilio (2012). El ejercicio de una ciudadanía responsable exige disponer de cierta competencia científica. En: Pedrinaci, Emilio. (Coord.). El desarrollo de la competencia científica. 11 ideas clave. Barcelona: Editorial GRAÓ

Perdomo, B. (2007). Aprendiendo a investigar dentro de un enfoque cooperativo: una experiencia educativa. Ponencia presentada en la vir Reunión Nacional de Currículum y i Congreso Internacional de Calidad e Innovación en Educación Superior. Sartenejas (Vol. 9). Recuperado de: http://www.saber.ula.ve/bitstream/123456789/38810/1/aprendiendo-investigar.pdf

Pozuelos, F. J. y G. Travé (2004). Aprender investigando, investigar para aprender: el punto de vista de los futuros docentes. Investigación en la Escuela, (54), 5-26. Recuperado de: http://www.investigacionenlaescuela.es/articulos/54/R54_1.pdf

Ramos, J. (2009). Enseñar a escribir con sentido. Revista Aula de Innovación Educativa, 185, 55-63. Recuperado de: http://s3.amazonaws.com/academia.edu. 
documents/43102306/AU18510-Estrategias-de-escritura-con-sentido.pdf?A WSAccessKeyId=AKIAIWOWYYGZ2Y53UL3A\&Expires=1500747263\&Sign

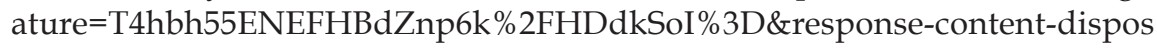
ition=inline \%3B\%20filename\%3DEnsenar_a_escribir_con_sentido.pdf

Rivera, Karen (2014). Éxito escolar en alumnado de enseñanza media superior. Un estudio de caso. Cádiz, Andalucía. En: Miranda, Carlos Federico; Pattaro, Fernanda y María Nohemí González. (Comps.). Género y ciencias sociales. Fronteras flexibles y fluidas. Colombia: Red HILA/Ediciones Universidad Simón Bolívar. 133-162.

Rizo, Marta (2006). Enseñar a investigar investigando. Experiencias de investigación en comunicación con estudiantes de la Licenciatura en Comunicación y Cultura de la Universidad Autónoma de la Ciudad de México. México: UACM. Recuperado de: http://test-departamento.pucp.edu.pe/comunicaciones/images/documentos/cap01-mrizo.pdf

Rodríguez, William; Hernández, Rubinsten; Muñoz, Liliana; Lizarazo-Camacho, Angélica María y Angélica Salamanca (2010). Actitudes hacia la ciencia: un campo de interés investigativo en la didáctica de las ciencias. Actualidades pedagógicas 57, 121-139. Recuperado de: http://revistas.lasalle.edu.co/index. php/ap/article/view/542/462

Rojas-Betancur, Mauricio y Raquel Méndez-Villamizar (2013). Cómo enseñar a investigar: Un reto para la pedagogía universitaria. Educación y Educadores, 16(1), 95-108. Recuperado de: http://www.scielo.org.co/pdf/eded/v16n1/ v16n1a06.pdf

Sánchez, Ricardo (1990). La vinculación de la docencia con la investigación. Una tarea teórica y práctica en proceso de construcción (el caso de la UNAM). Revista de la educación superior, (74), 5-50. Recuperado de: http:/ / publicaciones. anuies.mx/pdfs/revista/Revista74_S1A1ES.pdf

Sánchez, Ricardo (2014). Enseñar a investigar. Una didáctica nueva de la investigación en ciencias sociales y humanas. México: IISUE/UnAm. Recuperado de: file:/ / C:/Users/ IIDE/Downloads/787047082.01_ENSE\%C3\%91AR_A_INVESTIGAR.pdf

Sancho, Juana María (2001). Docencia e investigación en la universidad. Educar 28, 41-60. Recuperado de: http://ddd.uab.cat/pub/educar/0211819Xn28/02 11819Xn28p41.pdf

Soria, Óscar. Docencia de la investigación en la universidad larinoamericana ¿Por qué esperar hasta el posgrado? (2015). En: Morán, Porfirio (Comp.) Docencia e investigación en el aula. Una relación imprescindible. México: IISUE/UnAM.

Soto, Diego (2011). Investigar y publicar siendo estudiante de pregrado. Revista ANACEM, 5 (1), 70. Recuperado de: http://www.revistaanacem.cl/pdf/completos/vol51.pdf\#page $=75$

Trejo, María del Rosario y Fernando García (2006). Pedagogía de la investigación. Metodología de la Ciencia. Revista de la Asociación Mexicana de Metodología de la Ciencia y la investigación. 1 (1), 135-141. Recuperado de: http:/ /201.147.150.252:8080/xmlui/ bitstream/handle/123456789/1238/PedagogiaTrejoyCordoba.pdf?sequence $=1$

Wenger, Etienne (2010). Communities of practice and social learning systems: the career of a concept. En: Blackmore, Chris (Ed.) Social Learning Systems and communities of practice. UK: Springer 